\title{
TRANSFERENCIA DEL CONOCIMIENTO DE ENFERMERIA A LOS TUTORES INSTITUCIONALES EN EL CONTEXTO DE LA PRÁCTICA CLÍNICA
}

Martinez-Momblan, Ma Antonia, Colina-Torralva Javier, Basco-PradoLuis, Delgado-Hito, P, De la Cueva-Ariza Laura, Romero-García, M. Departamento de enfermería Fundamental y Medicoquirúrgica de la Universidad de Barcelona

\begin{abstract}
In the field of clinical practice, different agents intervene: the institutional tutor, academic tutor and coordinators of clinical practice subjects. It was proposed to train institutional tutors from specific and adequate training to ensure their training in the context of clinical practice subjects. OBJECTIVE. To evaluate the level of satisfaction of the institutional tutors with the training received in the context of the clinical practice of the Degree in Nursing Design. Descriptive and transversal. Population and sample. 162 institutional tutors during the academic year 2016-2017 who teach in the subjects of Clinical Stages I and I. Sampling for convenience. Instruments and procedure. To assess the level of satisfaction, an Ad-hoc Questionnaire with 5 dimensions was elaborated: Teaching, Methodology, Organization and facilities, Thematic contents and Satisfaction. The questionnaire had 14 items. The Microsoft Excel 2010 Program will be used as support. RESULTS. The final sample consisted of $90 \%(n=146)$ of the institutional tutors, with 93\% $(n=136)$ women. The overall level of satisfaction was 50.4, with minimum values of 38 and maximums of 56. CONCLUSIONS: The levels of overall satisfaction of the training received by the Institutional Tutors vary between satisfactory and very satisfactory.
\end{abstract}

Keywords: learning, nursing, students, practicum evaluation, satisfaction.

\section{Resumen}

En el ámbito de la práctica clínica intervienen diferentes agentes: el tutor institucional, tutor académico y coordinadores de asignaturas de práctica clínica. Se planteó formar a los tutores institucionales a partir de formación específica y adecuada para poder garantizar su formación en el contexto de las asignaturas de práctica clínica. OBJETIVO. Evaluar el nivel de satisfacción de los tutores institucionales con la formación recibida en el contexto de la práctica clínica del Grado de Enfermería Diseño. Descriptivo y 
TRANSFERENCIA DEL CONOCIMIENTO DE ENFERMERIA A LOS TUTORES INSTITUCIONALES EN EL CONTEXTO DE LA PRÁCTICA CLÍNICA

transversal. Población y muestra. 162 tutores institucionales durante el curso académico 2016-2017 que imparten docencia en las asignaturas de Estancias Clínicas I y I. Muestreo por conveniencia. Instrumentos y procedimiento. Para evaluar el nivel de satisfacción se elaboró un Cuestionario ad-hoc con 5 dimensiones: Docencia profesorado, Metodología, Organización e instalaciones, Contenidos temáticos y Satisfacción. El cuestionario contó con 14 ítems. Se utilizará como soporte el Programa Microsoft Excel 2010. RESULTADOS. La muestra final estuvo formada por el 90\% $(n=146)$ de los tutores institucionales siendo un 93\% $(n=136)$ mujeres. El nivel de satisfacción global fue de 50,4, con valores mínimos de 38 y máximos de 56. CONCLUSIONES: Los niveles de satisfacción global de la formación recibida por los Tutores Institucionales oscilan entre satisfactorios y muy satisfactorios.

Palabras clave: Aprendizaje enfermeria, estudiantes, evaluación, prácticas y satisfacción.

\section{Introducción}

En el ámbito de la práctica clínica intervienen diferentes agentes: el tutor institucional, tutor académico y coordinadores de asignaturas de práctica clínica. El Sistema Nacional de Innovación (SIN), establece que tan importante es generar el conocimiento, como difundirlo adecuadamente entre todos los agentes que lo conforman, siendo los tutores/as institucionales el nexo de unión entre el ámbito asistencial y el académico.

Los tutores institucionales se definen como aquellos enfermeros que participan en la formación de los estudiantes en las asignaturas de prácticas clínicas, a través de la evidencia y la reflexión de todas aquellas situaciones clínicas dando ayuda y soporte a la construcción 
Martinez-Momblan, Ma Antonia, Colina-Torralva Javier, Melero García Angeles, Fernández Cervilla Ana Belen, Delgado-Hito, P, De la Cueva-Ariza Laura, Romero-García, M.

del conocimiento. El tutor/a institucional se convierte en un elemento clave para apoyar la transferencia de conocimientos competenciales en la práctica clínica y promover una cultura de aprendizaje dentro de la organización $(1,2)$. Para asegurar la calidad e idoneidad del proceso formativo en las asignaturas de prácticas clínicas, es necesario que los tutores/as institucionales conozcan el marco general, normativa de la formación práctica, y actividades formativas y evaluativas necesarias para favorecer la calidad docente y mejorar el proceso enseñanza-aprendizaje del estudiante (3).

De acuerdo al Código Ético de las enfermeras y enfermeros de Cataluña, la formación de los estudiantes del Grado de Enfermería forma parte del compromiso profesional; así en su artículo 72 establece que "la enfermera colabora en la formación de los estudiantes de enfermería, creando un entorno propicio para el aprendizaje y transmitiendo los valores, conocimientos y habilidades propios de la profesión” (4).

La formación de las asignaturas de práctica clínica queda vinculada a la universidad ya que es quién define y articula el plan docente, selecciona los diferentes centros con convenio con la universidad donde los estudiantes realizaran sus periodos de prácticas clínicas y quién supervisa el desarrollo del proceso enseñanza aprendizaje del estudiante. Los centros que acogen a los estudiantes en prácticas juegan un papel principal en la formación de los estudiantes en prácticas, su seguimiento, realización de actividades formativas y la 
TRANSFERENCIA DEL CONOCIMIENTO DE ENFERMERIA A LOS TUTORES INSTITUCIONALES EN EL CONTEXTO DE LA PRÁCTICA CLÍNICA

evaluación de los resultados de aprendizaje en base a la adquisición de las competencias $(5,6)$.

Por todo lo dicho con anterioridad, se planteó formar a los tutores institucionales a partir de formación específica y adecuada para poder garantizar una formación de calidad en el contexto de las asignaturas de práctica clínica $(7,8)$ en las asignaturas de Estadas Clínicas I y II del Grado de Enfermería de la Facultad de Medicina y Ciencias de la Salud de la Universidad de Barcelona. Algunas evidencias consultadas, constatan la necesidad de mejorar su labor formativa a través de la formación especializada $(9,10)$.

\section{Objetivo}

Evaluar el nivel de satisfacción de los tutores institucionales con la formación recibida en el contexto de la práctica clínica del Grado de Enfermería de la Universidad de Barcelona.

\section{Desarrollo de la innovación}

En el contexto de la práctica clínica de los estudios de Ciencias de la Salud, intervienen diferentes agentes que deben adquirir conocimiento y habilidades que repercutiran directamente en la calidad de lo que hace y como lo hace. Es por ello, que la transferencia de conocimiento en el tutor institucional la hemos implementado a lo largo del año académico y en todos los centros que intervienen en la formación en prácticas clínicas de 
Martinez-Momblan, Ma Antonia, Colina-Torralva Javier, Melero García Angeles, Fernández Cervilla Ana Belen, Delgado-Hito, P, De la Cueva-Ariza Laura, Romero-García, M.

los estudiantes de la Escuela Universitaria de Enfermeria y dentro del Departamento de enfermería Fundamental y Medicoquirúrgica.

\section{Metodología}

Método. Metodología cuantitativa de tipo descriptivo y transversal.

Ámbito de estudio. La Escuela de Enfermería de la Facultad de Medicina y Ciencias de la Salud de la Universidad de Barcelona.

Población y muestra. 162 tutores institucionales de los siguientes centros sanitarios:

Consorci Sanitari Parc Taulí, Sant Joan Despí Moisés Broggi, Hospital de Viladecans, Fundació Esperit Sant, Sagrat Cor, Fundació Plató durante el curso académico 2016-2017 que imparten docencia en las asignaturas de Estancias Clínicas I y II del Grado de Enfermería. Muestreo por conveniencia.

Instrumentos y procedimiento. Para evaluar el nivel de satisfacción se elaboró un Cuestionario ad-hoc con 5 dimensiones y 14 ítems: Docencia profesorado; ¿Se han explicado con claridad?,¿Se han alentado la participación?. ¿Han facilitado el intercambio de experiencias? Y ¿Dominaba el tema a desarrollar. Metodología; ¿ha adquirod nuevos conocimientos?, ¿Se han cubierto los objetivos? Y ¿Ha sido adecuada la metodología alos objetivos planteados?.Organización e instalaciones; ¿La organización ha sido correcta?,¿La sala y/o instalaciones han sido adecuados? Y ¿El número de alumnos del grupo ha sido 
TRANSFERENCIA DEL CONOCIMIENTO DE ENFERMERIA A LOS TUTORES INSTITUCIONALES EN EL CONTEXTO DE LA PRÁCTICA CLÍNICA

adecuado?. Contenidos y temá; ¿En qué medida le han resultado útiles? ¿En qué medida se han cumplimentado las expectativas?. Satisfacción Global; ¿ ¿Grado de satisfacción con la teória impartida?, ¿Grado de satisfacción con la práctica impartida?. El cuestionario contó con 14 ítems y una pregunta abierta sobre posibles sugerencias en el contexto de la sesión recibida. Cada uno de los ítems se midió de acuerdo a una escala tipo Likert con 4 opciones de respuesta:1) Nada Satisfecho, 2) Poco Satisfecho, 3) Satisfecho, 4) Muy satisfecho, siendo la puntuación mínima de 14 y máxima de 56 (Tabla 1). También se recogieron variables sociodemográficas. La cumplimentación se realizó al finalizar las sesiones formativas a los tutores académicos de forma voluntaria y anónima. Dichas sesiones mostraron a los tutores académicos el marco normativo, las funciones del tutor/a académico, el marco competencial, los instrumentos de aprendizaje y la evaluación de los mismos, siempre desde la reflexión y evidencia en la práctica.

Tabla 1. Cuestionario ad-hoc satisfacción formación tutores institucionales

\begin{tabular}{|c|c|c|}
\hline $\begin{array}{c}\text { Dimensiones } \\
\text { de la escala }\end{array}$ & Nombre & Items del cuestinario \\
\hline 1 & Profesorado & 3 \\
\hline 2 & Metodología & 3 \\
\hline 3 & Organización/ & 2 \\
\hline 4 & Instalaciones & 2 \\
\hline 5 & Contenidos y temas & \\
\hline & Grado Satisfacción & \\
\hline
\end{tabular}

(cc) BY-NC-ND 2018, Universitat Politècnica de València Congreso IN-RED (2018) 
Martinez-Momblan, Ma Antonia, Colina-Torralva Javier, Melero García Angeles, Fernández Cervilla Ana Belen, Delgado-Hito, P, De la Cueva-Ariza Laura, Romero-García, M.

Aspectos éticos. Se solicitó el consentimiento informado a cada uno de los tutores participantes en las sesiones. Cuenta con la aprobación del Comité Bioético de la Universidad de Barcelona (IRB00003099).

Análisis de los datos. Análisis descriptivo mediante frecuencias y porcentajes e inferencial a partir del coeficiente de correlación de Spearman. Se utilizará como soporte el Programa Microsoft Excel 2010.

\section{Resultados}

La muestra final estuvo formada por el $90 \%(n=146)$ de los tutores institucionales siendo un 93\% (n=136) mujeres. El nivel de satisfacción global fue de 50,4, con valores mínimos de 38 y máximos de 56. En relación a las dimensiones analizadas se obtuvieron las siguientes medias de satisfacción: Docencia del profesorado de 3,9; Metodología 3,5; Organización e instalaciones 3,5; Contenidos Temáticos 3,6 y Satisfacción general 3,6. Las sugerencias muestran que el $37 \%(n=54)$ de los tutores institucionales necesita mayor información sobre el manejo de las rúbricas de evaluación (diario reflexivo, proceso enfermero, seminario de farmacología y seminario de nutrición) en el contexto de la práctica clínica y un 22\% $(n=32)$ refieren necesitar mayor formación a nivel general

\section{(Figura 2).}

(cc) EY-NC-ND 2018, Universitat Politècnica de València 


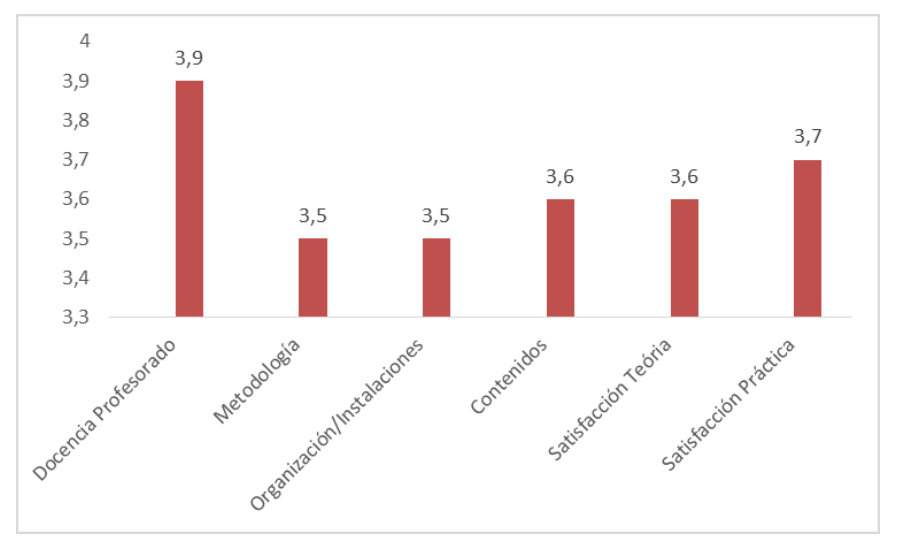

Figura 2. Satisfacción formación tutores institucionales

\section{Conclusiones}

Los niveles de satisfacción global de la formación recibida por los Tutores Institucionales oscilan entre satisfactorios y muy satisfactorios. Las sugerencias descritas nos orientan a acortar las distancias entre la tutora institucional y los responsables de asignaturas, estableciendo documentos marcos de práctica clínica que sirvan de guía y soporte en el proceso de seguimiento y evaluación de todos los agentes implicados en la formación de los estudiantes en el contexto de la práctica clínica.

\section{Bibliografía}

1.-Juvé Udina E, Farrero Muñoz S, Matad Calvo C, Monterde Prat D, Fierro Barrabás G, Marsal Serra R, Reyes Martin C, García Vidal B, Pons Prats A, Arnau Vives M, Martinez Luque $\mathrm{R}$, et al. ¿Cómo definen los profesionales de enfermería hospitalarios sus competencias asistenciales? Nursing 2007; 25 (7): 50-61. 
Martinez-Momblan, Ma Antonia, Colina-Torralva Javier, Melero García Angeles, Fernández Cervilla Ana Belen, Delgado-Hito, P, De la Cueva-Ariza Laura, Romero-García, M.

2.-Fahy A. Evaluating clinical competence assessment. Nurs Stand. 2011; 1723;25(50):42-48.

3.-Tanji S, Lopes CM, Vieira AP, Rodrigues W, De Paula C. Confrontación de criterios de evaluación del aprendizaje ante las apreciaciones de los estudiantes de enfermería. Enfermería Global. 2010; 9(1): 122-127.

4.-Judith Needham, Anne McMurray, Ramon Z. Shaban . Best practice in clinical facilitation of undergraduate nursing students. Nurse Education in Practice. 2016; 20: $131-138$.

5.-Kneafsey R. Developing skills in safe patient handling: mentors' views about their role in supporting student nurses. Nurse Educ Pract. 2007; 7(6):365-72.

6.-Ginsburg LR, Tregunno D, Norton PG. Self-reported patient safety competence among new graduates in medicine, nursing and pharmacy. BMJ Qual Saf. 2013; 22 (2): 147-54.

7.-Martínez Figueira, $M^{a}$ E. Ser tutor ¿cuestión de personalidad o de profesionalidad? Revista Española de Orientación y Psicopedagogía. 2010; 21 (3): 1-6.

8.-Molina, E. et al. La mejora del prácticum, esfuerzo de colaboración. Profesorado, Revista de Currículum y Formación de Profesorado. 2004; 8(2). 
TRANSFERENCIA DEL CONOCIMIENTO DE ENFERMERIA A LOS TUTORES INSTITUCIONALES EN EL CONTEXTO DE LA PRÁCTICA CLÍNICA

9.-Campbell,C.M., Smith,M., Dugan,J.P., and Komives, S.R. Mentors and college student

leadership outcomes: The importance of position and process. The Review of Higher

Education, 2012; 35(2): 595-625.

10.-Gershenfeld, S. A review of undergraduate mentoring programs. Review of

Educational Research, 2014; 84: 365-91.

(cc) EY-Nc-No 2018, Universitat Politècnica de València

Congreso IN-RED (2018) 